\title{
Random and externally controlled occurrences of Dansgaard-Oeschger events
}

\author{
Johannes Lohmann and Peter D. Ditlevsen \\ Centre for Ice and Climate, Niels Bohr Institute, University of Copenhagen, Copenhagen, Denmark \\ Correspondence: Johannes Lohmann (johannes.lohmann@nbi.ku.dk)
}

Received: 18 January 2018 - Discussion started: 9 February 2018

Accepted: 19 April 2018 - Published: 16 May 2018

\begin{abstract}
Dansgaard-Oeschger (DO) events constitute the most pronounced mode of centennial to millennial climate variability of the last glacial period. Since their discovery, many decades of research have been devoted to understand the origin and nature of these rapid climate shifts. In recent years, a number of studies have appeared that report emergence of DO-type variability in fully coupled general circulation models via different mechanisms. These mechanisms result in the occurrence of DO events at varying degrees of regularity, ranging from periodic to random. When examining the full sequence of DO events as captured in the North Greenland Ice Core Project (NGRIP) ice core record, one can observe high irregularity in the timing of individual events at any stage within the last glacial period. In addition to the prevailing irregularity, certain properties of the DO event sequence, such as the average event frequency or the relative distribution of cold versus warm periods, appear to be changing throughout the glacial. By using statistical hypothesis tests on simple event models, we investigate whether the observed event sequence may have been generated by stationary random processes or rather was strongly modulated by external factors. We find that the sequence of DO warming events is consistent with a stationary random process, whereas dividing the event sequence into warming and cooling events leads to inconsistency with two independent event processes. As we include external forcing, we find a particularly good fit to the observed DO sequence in a model where the average residence time in warm periods are controlled by global ice volume and cold periods by boreal summer insolation.
\end{abstract}

\section{Introduction}

During the last glacial period, lasting from approximately 120 to $12 \mathrm{kyaBP}$ (thousands of years before present), a large number of abrupt large-scale climate changes have been recorded in Greenland ice cores and other Northern Hemisphere climate proxies. These so-called DansgaardOeschger (DO) events (Dansgaard et al., 1993) are characterized by an abrupt warming of $10-15 \mathrm{~K}$ from cold conditions (stadials) to warmer conditions (interstadials) within a few decades. This is typically followed by gradual cooling, lasting centuries to thousands of years, until a more abrupt jump back to cold conditions is observed. The warming events are not regularly spaced over the glacial, but rather distributed in a complex temporal pattern, as can be seen in the NGRIP ice core record in Fig. 1. This raises questions about the causes of these recurring climate changes. Could an internal oscillation of large components of the climate system under strongly varying conditions give rise to this pattern? Or, in contrast, are the climate changes manifestations of highly sensitive, multi-stable climate system components, where jumps in between different states are triggered in an unpredictable way by one or possibly many different other chaotic components?

Since the discovery of these unexpected climate events with no known cause, questions of this kind have been addressed. Whereas high-resolution coupled climate models under glacial conditions typically lack DO-type variability, models of intermediate complexity and simpler conceptual models have been proposed to explain qualitative features of the sequence of last glacial climate changes. Starting from the discovery of an approximate 1500-year spectral signature in the GISP2 ice core record (Grootes and Stuiver, 1997) and an apparent in-phase pacing of individual events by mul- 


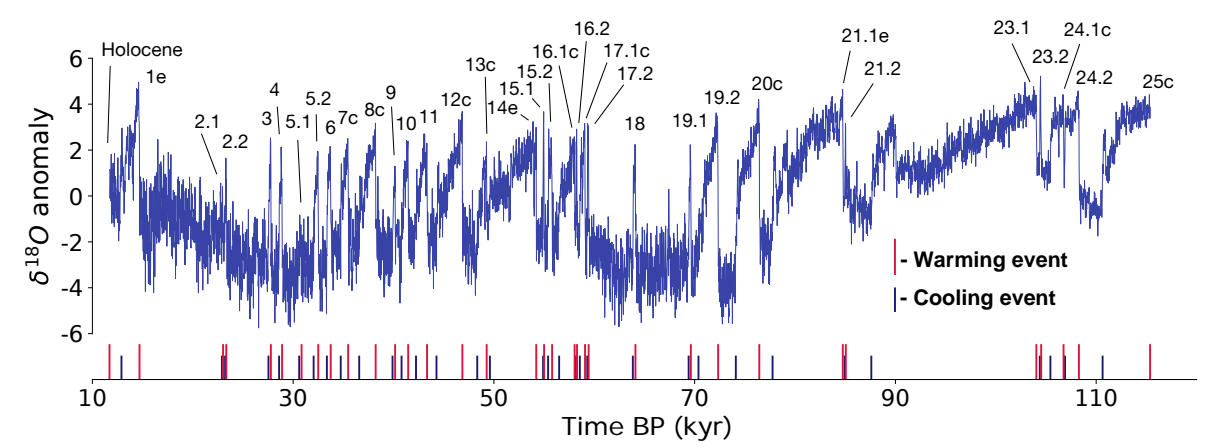

Figure 1. NGRIP oxygen isotope ice core record in 20-year binned resolution and associated Dansgaard-Oeschger warming and cooling events. The numbers above the time series indicate the warming transitions into the respective Greenland interstadials. The nomenclature is adopted from Rasmussen et al. (2014), and only events considered in this study are marked. On the time axis we marked the timing of warming (red) and cooling (blue) events.

tiples of this time period (Alley et al., 2001; Schulz, 2002; Rahmstorf, 2003), a number of competing hypotheses have been compared to the data. Among these are studies aiming to establish a mechanism for this periodicity, including direct triggering by periodic forcing (Braun et al., 2005), stochastic resonance (Alley et al., 2001), ghost resonance (Braun et al., 2007) and coherence resonance (Timmermann et al., 2003). On the other hand, it has been shown that there is limited significance to the periodic spectral signature (Braun et al., 2010) and pacing of individual events (Ditlevsen et al., 2007). When including data reaching further back in time than 50 kya BP it is found that only very weak periodic contributions to modeled switching sequences are compatible with the data and that instead it is more likely that the observed sequence of events is a realization of a purely noisedriven process (Ditlevsen et al., 2005).

In this work, we want to expand on this idea by testing whether the observed sequence of events is indeed consistent with one or more random, stationary processes, or whether the changes over time of the properties of the observed event sequence require modulating parameters of the governing process over time. To this end, we consider the whole glacial period, as opposed to previous efforts focusing on a rather regular period in the middle of the glacial. We investigate two different levels in detail of description by first only regarding the sequence of warming events and second the combined sequence of alternating transitions in between cold and warm conditions. We proceed by testing two null hypotheses: (1) the sequence of DO warming events is a realization of a Poisson process with fixed rate parameter; (2) the sequence of stadials and interstadials is a realization of two independent Poisson processes with fixed rate parameters giving rise to transitions in between stadials and interstadials. In order to test the hypotheses, we consider the evolution of the number of warming events in a moving window of $20 \mathrm{kyr}$. This quantity measures how variable the average event frequency is over time, a property which we denote as irregularity, and in the DO sequence it deviates strongly from a constant occur- rence frequency of events over time. We test whether samples from the abovementioned stationary processes show similar irregularity.

In addition to the evolution of the frequency of warming events we look at the evolution of the abundance of the stadial over the interstadial condition, which changes significantly over time in the DO sequence. This additional nonstationary structure in the data is the basis for another hypothesis test we perform. Finally, we test how the models' support with respect to the data is improved as we force the rate parameters with a combination of a global climate proxy and orbital variations of insolation to incorporate changing background climate conditions. The main findings of this study are as follows: (1) a Poisson process with fixed rate parameter, modeling warming transitions only, is consistent with the time variations in the NGRIP DO warming event sequence; (2) a model composed of two independent stationary Poisson processes governing transitions in between stadials and interstadials is not consistent with the time variations in the observed DO event sequence; (3) forcing the aforementioned models with a combination of a global ice volume proxy and a summer insolation curve leads to good statistical agreement with the observed sequence. Specifically, we find good agreement for a model with two individual processes, where the average transition rate from interstadial to stadial is controlled by global ice volume forcing, obtained from independent ocean core isotope records, and the average transition rate from stadial to interstadial is controlled by boreal summer insolation.

The paper is structured in the following way. In Sect. 2 we introduce in more detail the data used in this study, the summary statistics used to investigate irregularity in the event series, the models used to explain the data and the hypothesis test procedure. In Sect. 3 we present the results of the hypothesis tests on the different models. We discuss and interpret the results in Sect. 4. 


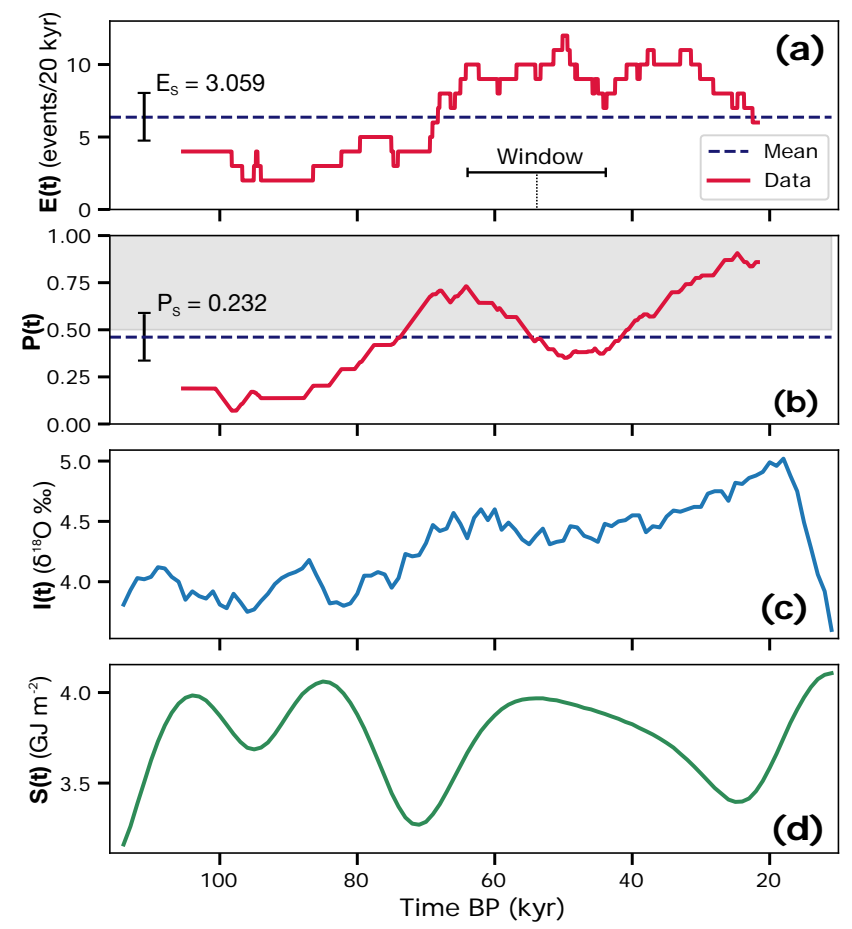

Figure 2. Time-varying irregularity indicators calculated from the NGRIP DO sequence, and climate forcings. (a) The number of warming events in a running $20 \mathrm{kyr}$ window $E(t)$ (red) and the mean value (dashed blue). (b) The abundance of stadials in a $20 \mathrm{kyr}$ window $P(t)$. For values greater than 0.5 (indicated by gray shading) the portion of stadials is larger than the portion of interstadials within the window. (c) Ocean sediment proxy record for global ice volume $I(t)$. (d) Integrated summer insolation at $65^{\circ} \mathrm{N} S(t)$.

\section{Methods and models}

Our study of the sequence of DO events is based on the refined dating represented by the GICC05 timescale (Svensson et al., 2006), the classification of Greenland stadials (GS) and Greenland interstadials (GI) given in Rasmussen et al. (2014) and the timings reported therein. We consider all stadials and interstadials and corresponding transitions, starting with GI$25 \mathrm{c}$ at $115370 \mathrm{kyaBP}$ and ending with the transition from GS-1 to the Holocene at 11703 kya BP. We do not include events classified as sub-events, i.e., drops in the middle interstadials to colder, but not fully stadial conditions, with the exception of GS-14. This yields a total number of 34 warming events and 33 cooling events. This increase in number from the 25 originally reported warming events is due to refined subdivision (Rasmussen et al., 2014).

Given sequence and timing of transitions in between stadials and interstadials, we construct time-varying indicators of irregularity in the sequence of event timings, which are shown in Fig. 2a and b. To this end, we calculate the number of warming transitions within a moving window of $20 \mathrm{kyr}$ at midpoint in time $t$, which we denote as $E(t)$. The window size of $20 \mathrm{kyr}$ is chosen as trade-off between resolution and statistical robustness of longer-term features in the event sequence given the characteristic timescale of event occurrence of $3.1 \mathrm{kyr}$. The window is furthermore of comparable size to dominant variations in global background climate and insolation forcing, which will be investigated below. We obtain a time series indicating the deviation in the occurrence frequency of warming events from an evenly spaced (regular) event occurrence. We summarize this in a scalar test statistic $E_{\mathrm{S}}$ defined as the root mean squared deviation of the time series $E\left(t_{n}\right)$ from the expectation value $\bar{E}$

$E_{\mathrm{S}}=\sqrt{\left.\frac{1}{N} \sum_{n=1}^{N}\left(E\left(t_{n}\right)-\bar{E}\right)^{2}\right)}$,

where $\bar{E}=6.367$ is the average number of events per $20 \mathrm{kyr}$ of the whole DO sequence. For events occurring periodically with a period significantly smaller that the window size, the test statistic $E_{\mathrm{S}}$ is close to zero. For completely randomly occurring events the test statistic shows a finite value, which depends on the moving window size relative to the average waiting time in between events. The same time-varying indicator has previously been used to complement a model comparison study aiming to quantify the influence of external forcing to conceptual models of the NGRIP ice core record (Mitsui and Crucifix, 2017). With this statistic we test whether the observed DO sequence departs significantly further from regularity as compared to what is expected by a random, uncorrelated event sequence. If this is the case, it would hint either at non-stationarity of the underlying process or a super-exponential event waiting time distribution. We consider the latter scenario to be less likely since no clear motivation such a process exists.

While no significant correlation between duration of individual stadials and preceding or subsequent interstadial is observed (Pearson's $r=0.04$ and $r=-0.15$, respectively), the data suggests long-term variations in stadial and interstadial duration distributions. If these variations are systematic for stadials and interstadials (i.e., correlated or anticorrelated) they should be detectable in the correlation of individual neighboring stadial/interstadial durations given a large enough sample size. However, due to the small sample size of events in this study and the broad distribution of event waiting times a correlation due to long-term trends is not observed in practice. It is thus necessary to devise another time-varying indicator in order to capture additional detail in the structure of the DO sequence. When observing a given number of events in a time window, this may be either comprised of a combination of long stadials and short interstadials, or short stadials and long interstadials. This is not resolved in the statistic $E_{\mathrm{S}}$. To capture this structure, we investigate the total portion of stadials within a moving window. Given the sum of the duration of all stadials $T_{\mathrm{st}}(t)$ in a time window around a given midpoint in time $t$, the indicator is defined as $P(t)=T_{\mathrm{st}}(t) \cdot(20 \mathrm{kyr})^{-1}$. We summarize this 
indicator with the scalar test statistic $P_{\mathrm{S}}$. It is defined as the root mean squared deviation from the average value $\bar{P}$

$P_{\mathrm{S}}=\sqrt{\left.\frac{1}{N} \sum_{n=1}^{N}\left(P\left(t_{n}\right)-\bar{P}\right)^{2}\right)}$,

where $\bar{P}=0.461$ is the sum of all stadial durations divided by the total duration of the last glacial period.

We now describe the models which are used to evaluate our hypotheses on the data using the test statistics described above. The first model used in our study models the process generating the sequence of warming events as a Poisson process with fixed rate parameter $\lambda$, i.e., we disregard the cooling transitions in between warming events. It is denoted as "oneprocess model" hereafter. The inverse of the rate parameter corresponds to the average waiting time in between warming events. The Poisson process corresponds to a situation where there is no memory of the past and thus the probability for a transition is determined by $\lambda$ and is independent of time. All information on climate stability is represented in the parameter $\lambda$. We set its value equal to the inverse of the empirically observed average waiting time over the entire glacial record. This yields $\lambda=(3.141 \mathrm{kyr})^{-1}$.

As a second model, labeled "two-process model" hereafter, we propose two individual processes for generating warming transitions from stadials to interstadials and cooling transitions from interstadials back to stadials. Each is represented by a Poisson process with a fixed rate $\lambda_{1}$ and $\lambda_{2}$, for warming and cooling, respectively. Again, the parameters are derived from the data by considering the empirical average residence times in stadials and interstadials, yielding $\lambda_{1}=(1.477 \mathrm{kyr})^{-1}$ and $\lambda_{2}=(1.663 \mathrm{kyr})^{-1}$. The model is different from the one previously introduced in that the sequence of warming transitions is not a Poisson process, but a more regular one that is obtained from the sum of two independent processes. The probability distribution of waiting times $T$ in between warming events is not exponential, but can be evaluated, yielding

$P(t>T)=\left(\lambda_{1}-\lambda_{2}\right)^{-1} \cdot\left(\lambda_{1} e^{-\lambda_{2} \mathrm{~T}}-\lambda_{2} e^{-\lambda_{1} \mathrm{~T}}\right)$.

The average interstadial and stadial durations of the data seem to behave differently over the course of the glacial, as captured by our second test statistic. This motivates us to study whether this behavior is likely to be encountered by chance assuming randomness and independence of both warming and cooling transitions.

As comparison to our hypothesis of stationary random processes, we consider the same models with time-varying rate parameters, which are given by a linear combination of two external climate factors: $\lambda=\widetilde{\lambda}+a S(t)+b I(t)$. Firstly, we use a measure of incoming solar radiation at $65^{\circ} \mathrm{N}$ integrated over the summer $S(t)$ (Huybers, 2006). It is defined as the annual sum of the insolation on days exceeding an average of $350 \mathrm{~W} \mathrm{~m}^{-2}$. Secondly, we use the LR04 ocean sediment record stack as proxy for global ice volume $I(t)$ (Lisiecki and Raymo, 2005). We note that, in contrast to insolation, global ice volume is not an external factor in the strict sense. However, its dominant variability is on longer timescales than DO events and most importantly it is obtained from an independent data source. Time series of these forcings are shown in Fig. 2c and d. The models' parameters are chosen such that the time-varying indicators are on average closest to those of the data. Specifically, using Monte Carlo simulation we generate many realizations for a fixed model parameter, compute time-varying indicators for each realization and then construct an average curve. Finally, the root-mean-square deviation (RMSD) from this curve with respect to the timevarying data statistic is computed. For best fit, we search for the least RMSD on a grid in parameter space. This corresponds to a numerical calculation of the maximum likelihood fit to the observed data. The two-process model is fitted to $E(t)$ and $P(t)$ simultaneously, by minimizing the normalized

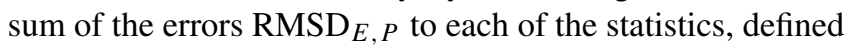
as $\mathrm{RMSD}_{\text {sum }}=\mathrm{RMSD}_{P} / E_{\mathrm{S}}+\mathrm{RMSD}_{P} / P_{\mathrm{S}}$.

The hypothesis tests are performed in the following way. For a given model we simulate a large number of realizations, which are collections of subsequent events with the same total duration as the record (104 kyr). For each realization we calculate the time-varying indicator of interest and the corresponding scalar test statistic. We then use the distribution of test statistics for a one-sided hypothesis test. The test simply counts how many test statistics in the ensemble are as large as or larger than the test statistic obtained from the data. Divided by the sample size, this yields a $p$ value, which estimates the probability of generating a random realization under the null hypothesis model that is at least as extreme as the observed data. We can reject the null hypothesis at a confidence level $\alpha$ if the $p$ value is smaller than $1-\alpha$.

\section{Results}

The results of the hypothesis test on the stationary one- and two-process models are shown in Fig. 3a-c. The plots show test statistics distributions of the respective null models and the corresponding test statistic value of the data. For the one-process model, the data test statistic lies well within the distribution, yielding a $p$ value of $p_{E}=0.16$, as seen in Fig. 3a. Thus, we cannot rejected the null hypothesis at a level $>85 \%$. This indicates that the variations in the timing of warming transitions are consistent with a stationary Poisson process, i.e., without invoking variations in the rate parameter. Figure $3 b$ and $c$ show the hypothesis tests of the two-process model, yielding low values of $p_{E}$ and $p_{P}$ for both test statistics. The stationary two-process model is thus rejected by the hypothesis tests with both test statistics at high confidence $>98 \%$.

To better visualize the outcomes of the hypothesis tests, we show confidence bands for the time-varying indicators 

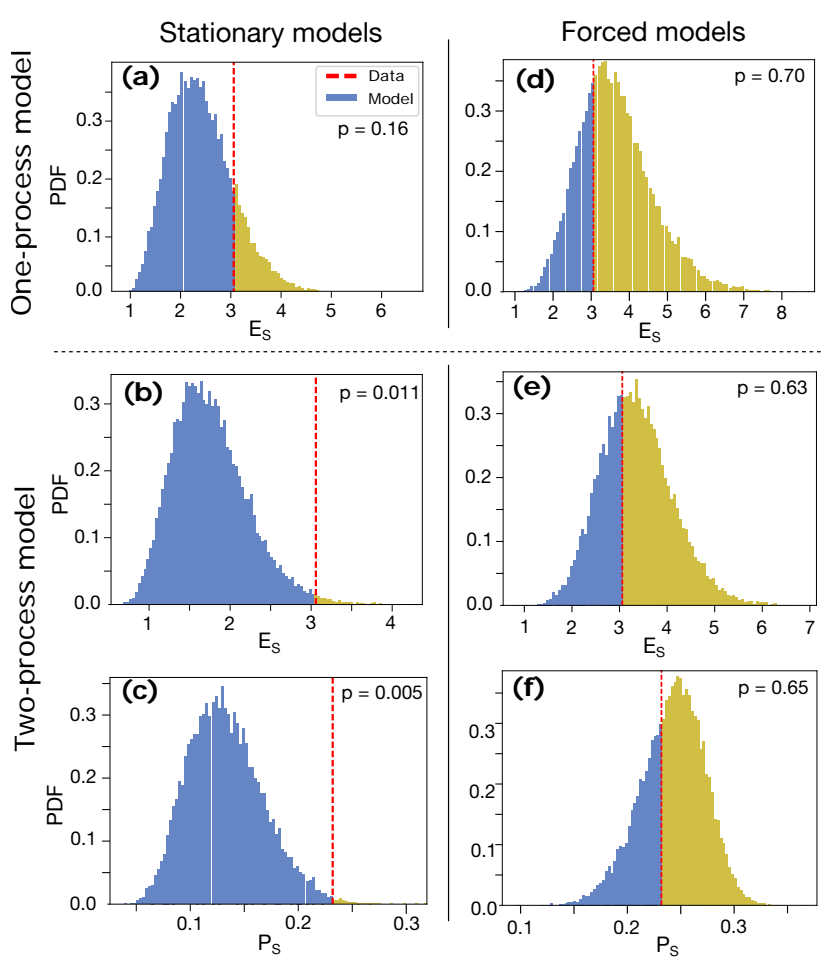

Figure 3. Empirical distributions from Monte Carlo simulation of the test statistics for the stationary $(\mathbf{a}-\mathbf{c})$ and the forced null model (d-f). The test statistics $E_{\mathrm{S}}$ of the one-process models are shown in panels (a) and (d). The test statistics $E_{\mathrm{S}}$ and $P_{\mathrm{S}}$ of the two-process models are shown in panels (b) and (e), and (c) and (f), respectively. The position of the data test statistic within the distribution is marked in red and determines the $p$ value of the hypothesis tests.

from our Monte Carlo simulations in Fig. 4. The indicator $E(t)$ of the data lies within the $95 \%$ point-wise confidence band of the one-process model. Moreover, this band can be calculated analytically due to the fact that the probability distribution of observing $k$ events in a time period $T$ is given by the Poisson distribution $P(k, T)=\frac{(\lambda T)^{\mathrm{k}}}{k !} e^{-\lambda \mathrm{T}}$. The cumulative distribution thereof allows us to calculate the probabilities of observing the minimal and maximal number of events per $20 \mathrm{kyr}$ found in the data indicator $E(t)$. We find the probability to observe 2 or less events is $P=0.047$ and to observe 12 or more events $P=0.030$. This confirms that we cannot exclude the possibility of observing only 2 events or as much as 12 events during $20 \mathrm{kyr}$ of the record at $95 \%$ confidence. The $95 \%$ confidence band of $E(t)$ for the two-process model in Fig. $4 \mathrm{~b}$ is narrower and does not include the most extreme parts of the data curve. The same holds for the indicator $P(t)$, thus confirming that the two-process model can be ruled out with high confidence as null model for the observed sequence of events.

In the following we present the hypothesis tests performed on the one- and two-process models forced with insolation $S(t)$ and ice volume $I(t)$, which are both scaled to zero mean

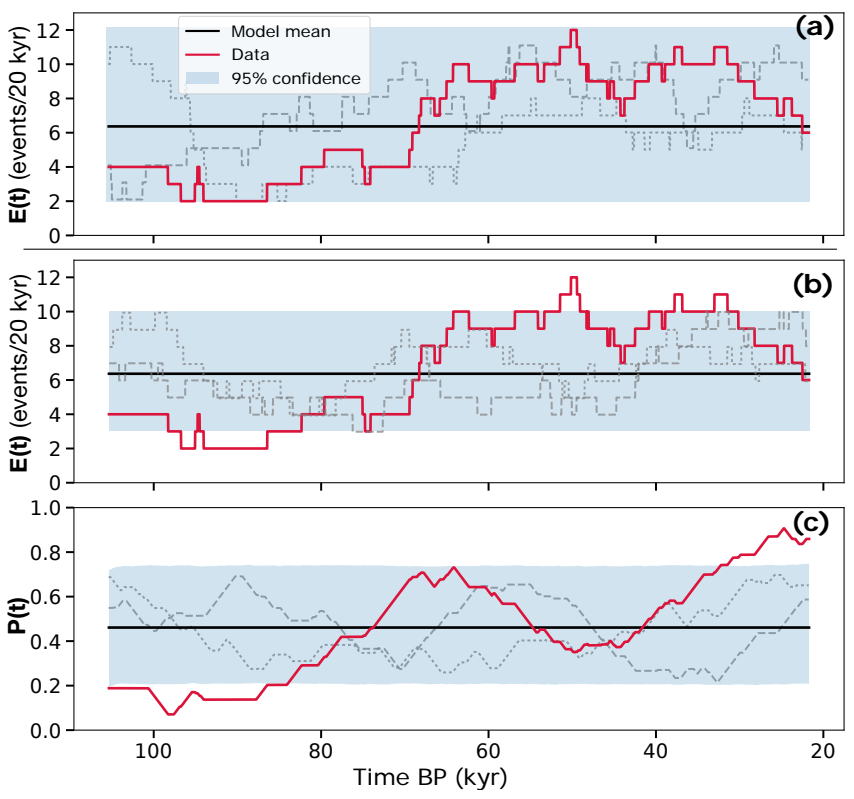

Figure 4. Point-wise $95 \%$ confidence bands and model mean (black line) for the time-varying indicators $E(t)$ and $P(t)$ from Monte Carlo simulations. (a) $E(t)$ for the stationary one-process model. (b) $E(t)$ and (c) $P(t)$ for the stationary two-process model. The indicators for the data are shown in red and two typical model realizations are shown in gray.

and range 1. Figure 5 shows the time-dependent transition rates as obtained from the parameter fit. For the one-process model we obtain

$\lambda(t)=0.32+0.43 \cdot S(t)+0.82 \cdot I(t)$.

The best-fit two-process model has warming transition rate $\lambda_{1}(t)$ and cooling transition rate $\lambda_{2}(t)$ :

$\lambda_{1}(t)=0.97+1.60 \cdot S(t)-0.57 \cdot I(t)$,

$\lambda_{2}(t)=0.97-1.96 \cdot S(t)+2.56 \cdot I(t)$.

The hypothesis tests for the fitted models are shown in Fig. 3d-f and yield high $p$ values, where the data statistic lies near the mode of the distributions. Note that we only measure the deviation of the time-varying statistics from a constant average value. Thus, the statistical test is not targeted at evaluating the fit to the data, but merely at probing whether the fluctuations over time of the indicators are of the right magnitude. Goodness of fit can be seen via the means of the confidence bands and mean of the time-varying indicators, as shown in Fig. 6. For both models, the mean indicators lie close to the data curves, which consequently lie within $95 \%$ confidence bands. We summarize all model parameters, hypothesis test results and goodness-of-fit values in Table 1.

We additionally report how the goodness of fit of the forced models changes when using only partial forcing and 
Table 1. Summary of model parameters, hypothesis test results and goodness of fit of the mean model time-varying indicators with respect to the data.

\begin{tabular}{llll}
\hline Model & Parameters & $p$ value & Goodness of fit \\
\hline Stationary one-process & $\lambda=0.32$ & $p_{E}=0.16$ & RMSD $=3.05$ \\
\hline \multirow{2}{*}{ Stationary two-process } & $\begin{array}{l}\lambda_{1}=0.68 \\
\lambda_{2}=0.60\end{array}$ & $\begin{array}{l}p_{E}=0.011 \\
p_{P}=0.005\end{array}$ & RMSD $_{\text {sum }}=2.0$ \\
\hline \multirow{2}{*}{ Non-stat. one-process } & $\tilde{\lambda}=0.32$ & $p_{E}=0.70$ & RMSD $=1.42$ \\
& $a=0.43, b=0.82$ & & \\
\hline \multirow{2}{*}{ Non-stat. two-process } & $\tilde{\lambda}_{1}=0.97, \tilde{\lambda}_{2}=0.97$ & $p_{E}=0.63$ & RMSD $_{\text {sum }}=0.59$ \\
& $a_{2}=-1.60, b_{1}=-0.57$ & $p_{P}=0.65$ & \\
\hline
\end{tabular}

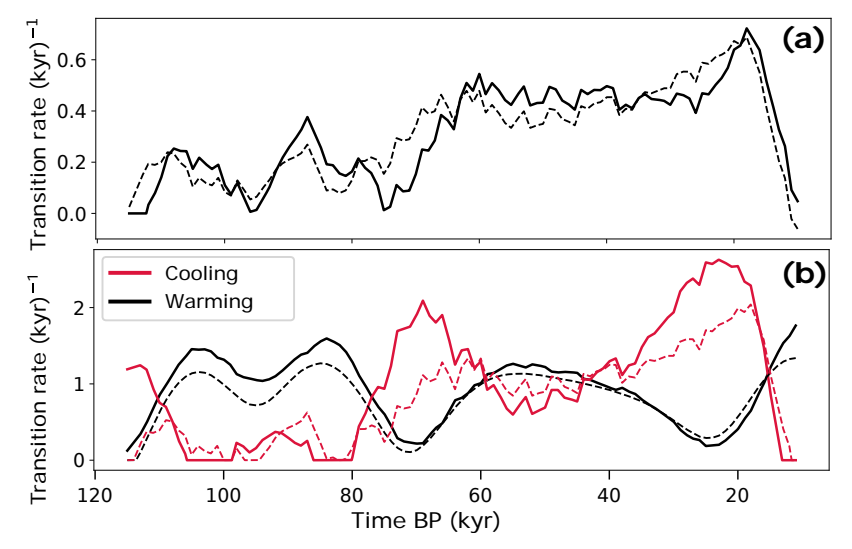

Figure 5. Time-varying transition rate parameters of the best-fit one-process $\lambda(t)$ (a) and two-process (b) models $\lambda_{1}(t)$ and $\lambda_{2}(t)$, as well as of the reduced models (dashed lines).

thus a reduced number of parameters. When forcing the oneprocess model with both ice volume and insolation, we yield an RMSD of the model mean $E(t)$ from the data curve of 1.42. Forcing with ice volume (insolation) only yields a bestfit RMSD of 1.64 (3.00). As baseline comparison, the RMSD from the unforced model to the data curve is equal to $E_{S}$; i.e., it has an RMSD of 3.05. The model forced with ice volume fits the data only marginally worse than the model with both forcings and for comparison we show the mean timevarying indicator $E(t)$ for this model in Fig. 6a with a green dashed curve. For the two-process model, we considered all combinations where both warming and cooling processes are only forced by either ice volume or insolation. Goodness of fit in the two-process model is given by the sum of errors of both indicators $\mathrm{RMSD}_{\text {sum }}$. For the best-fit model with full forcing we find $\mathrm{RMSD}_{\text {sum }}=0.59$, whereas the baseline of an unforced model gives $\mathrm{RMSD}_{\text {sum }}=2.0$. Forcing both warming and cooling processes with ice volume (insolation) only yields a best-fit of $\mathrm{RMSD}_{\text {sum }}=0.90$ (1.60). Using insolation for the warming transitions and ice volume forcing for the cooling transitions yields $\mathrm{RMSD}_{\text {sum }}=0.68$, while the
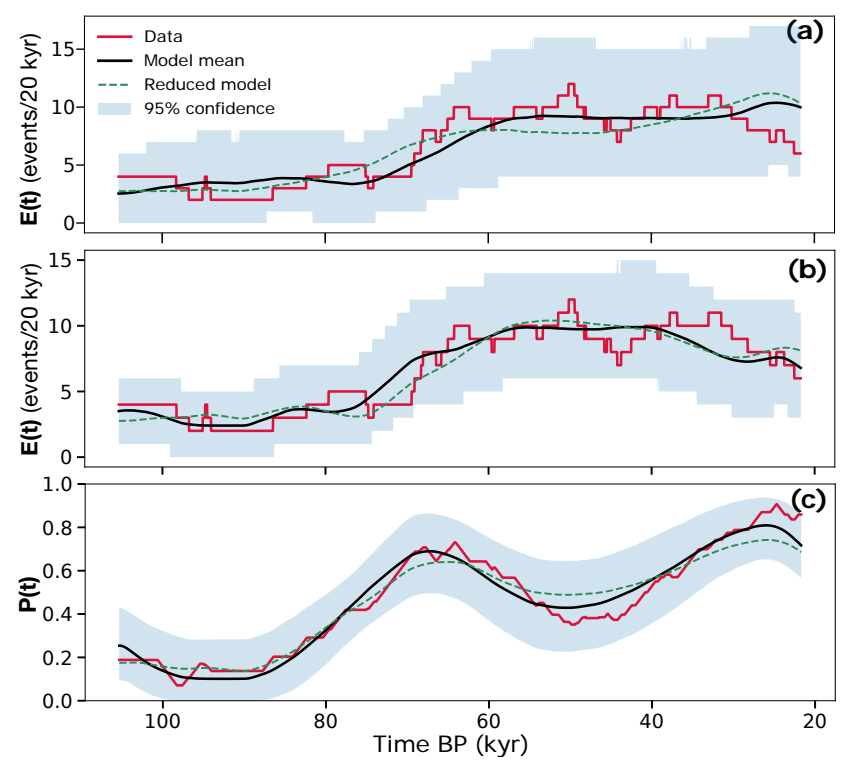

Figure 6. Point-wise $95 \%$ confidence bands and model mean (black curve) for the time-varying indicators $E(t)$ and $P(t)$ from Monte Carlo simulations. (a) $E(t)$ for the best-fit non-stationary one-process model with full forcing. (b) $E(t)$ and (c) $P(t)$ for the best-fit non-stationary two-process model with full forcing. The indicators for the data are shown in red. The green dashed line indicates the best-fit model mean curves for the respective models with reduced forcing, as described in the main text.

converse choice yields $\mathrm{RMSD}_{\text {sum }}=1.68$. Thus, the only reduced two-process model yielding a comparable goodness of fit compared to the model with full forcing is the model with insolation forcing on warming transitions and ice volume forcing on cooling transitions. It is defined by

$\lambda_{1}(t)=0.81+1.54 \cdot S(t)$,

$\lambda_{2}(t)=0.80+2.39 \cdot I(t)$.

We show the mean time-varying indicators for this model in Fig. $6 \mathrm{~b}$ and $\mathrm{c}$ with a green dashed curve. 


\section{Discussion and conclusions}

Our first result considers only the warming events. While the distribution of waiting times in between warming events is well modeled by an exponential distribution (not shown here), we show that the number of events in a moving window of $20 \mathrm{kyr}$ (and thus the mean waiting time) clearly changes over time, but no more than would be expected from a realization of a stationary Poisson process. Thus, if there is a unique process giving rise to the warming transitions, it need not be changing over time due to external factors. Although the description of DO events solely by the timing of the abrupt warming is very simplistic, we still think it is a useful result since the abrupt warming events are the most robust feature in ice cores and other proxy records and are commonly used to assess synchronicity and pacing of abrupt climate change in the last glacial.

The second result indicates, however, limits to the stationarity in the sequence of events as we increase the detail of description. Assuming two independent processes giving rise to transitions from stadials to interstadials and vice versa, the null hypothesis of stationarity can be rejected with both our statistics. Specifically, both the variations over time of the number of warming events and the relative durations of stadials and interstadials are too large to be consistent with our two-process model using constant parameters. This model gives rise to a more regular sequence of warming events, compared to the one-process model. This is because one DO cycle is the sum of two independent processes and thus its duration does not follow an exponential distribution (coefficient of variation $\mathrm{CV}=1.0$ ), but rather Eq. (3), which is less dispersed $(\mathrm{CV}=0.708)$. In the limiting case of a DO cycle comprised of a very large sequence of $N$ independent and stationary processes, one finds a Gaussian distribution of waiting times with decreasing variance as $N$ grows. This then corresponds to an almost evenly spaced sequence of events, which is not supported by the observations.

Next, we investigated improvements of the consistency of the models with the data by allowing their parameters to vary over time as linear combination of two climate forcings. Choosing the best-fit linear combination of forcings, we found the average time-varying indicators of both models to match very well to the data curve. Thus, whereas the data were seen as a rather out-lying realization consistent with a one-process model but not with a two-process model, when introducing forcings the data become the expected behavior of the models. The goodness of fit follows from the correlation of the time-varying indicators and the forcings, which can be seen in Fig. 2. For the ice volume proxy we find a Pearson correlation of $r^{2}=0.78$ with $P(t)$ and $r^{2}=0.58$ with $E(t)$. We assess the significance of this correlation by fitting an $\mathrm{AR}(1)$ process to the linearly detrended ice volume and conduct a hypothesis test yielding a correlation with $P(t)$ of 0.33 with a $p$ value of $p=0.035$ and thus significance at $95 \%$ confidence. In contrast, the correlation of the indicator
$E(t)$ and ice volume does not go beyond the linear trend. We do not assess the significance of correlations of insolation with the time-varying indicators, since it is difficult to find a good null model in this case.

Finally, we discuss the importance of ice volume and insolation in the best-fit one- and two-process models. In the oneprocess model, Eq. (4) shows that both increased insolation and ice volume lead to higher occurrence rates of events, with the contribution of ice volume approximately twice as large as that of insolation. We note that the decrease in DO activity towards the Last Glacial Maximum (LGM) is not captured by the model because the ice volume forcing is dominating. The individual contributions in the best-fit model do not completely capture the importance of the two forcings. There are directions in the likelihood landscape of parameters which are very flat. As a result, we found that the best-fit model with only ice volume forcing yields a fit only marginally worse than the best-fit model with both forcings. We thus conclude that ice volume is clearly the more important control on the sequence of warming events. This is consistent with the findings in Mitsui and Crucifix (2017), where Bayesian model selection criteria show that global ice volume is a more important forcing than insolation in stochastic dynamical systems as models for Greenland ice core records.

In the two-process model the warming and cooling transition rates are influenced by the forcing in opposite ways, as can be seen from Eq. (5): warming transitions from stadials to interstadials become more likely for higher insolation and lower ice volume, and vice versa for cooling transitions from interstadials to stadials. For cooling transitions, the contribution of ice volume is slightly larger than that of insolation. The warming transition rate is dominated by insolation, which contributes three times more than ice volume. With this model, the overall trend of mean waiting times in between warming events and of the stadial abundance is well captured, including the decrease in activity towards the LGM. Similar to the one-process model, we found a more parsimonious model which fits the data almost as well as the best-fit model with full forcing. This model uses only insolation forcing for stadials and ice volume forcing for interstadials, which complements the analysis of the individual contributions in the fully forced model. We thus hypothesize that based on our study there is evidence for insolation control on average stadial duration and ice volume control on average interstadial duration. This finding could hint at two distinct mechanisms responsible for transitions in between regimes.

An exhaustive investigation of whether our model description and subsequent findings are consistent with governing mechanisms for DO-type variability inferred from detailed data and realistic model studies is beyond the scope of this paper. Nevertheless we conclude the discussion with some interpretations which are more speculative in nature. We begin with insolation control on stadial duration. Boreal summer insolation might influence the occurrence frequency of warming transitions by modulating the ice-ocean albedo 
feedback, which amplifies break-up or export of larger areas of sea ice. Sea ice decrease could subsequently cause rapid warming through subsurface ocean heat release (Dokken et al., 2013). Initial openings of the sea ice cover might be created by wind stress. Evidence for stochastic wind stress forcing and subsequent sea ice changes have been reported in unforced model studies of rapid climate transitions (Drijfhout et al., 2013; Kleppin et al., 2015). To explain global ice volume control on interstadial duration we invoke different influences on the strength and stability of the interstadial (strong) mode of the Atlantic Meridional Overturning Circulation (AMOC). If we consider global ice volume as an indicator of mean global climate, we find consistency with coupled climate simulations that show correlation of the stability of the strong AMOC branch to freshwater hosing and mean climate state (Kawamura et al., 2017). We furthermore note the study in Buizert and Schmittner (2015), where a correlation of individual interstadial duration and Antarctic temperatures from ice cores is established and explained by influences of Southern Ocean processes on the strength and stability of the AMOC. Given the strong similarity of the global ice volume record and Antarctic ice core records on longer timescales, this is closely related to our findings. We finally note that in our model description, the trigger for warming and cooling events is stochastic and thus different from nearperiodic DO cycles (Peltier and Vettoretti, 2014).

In conclusion, we show that the long-term variations in DO warming event frequency, often described as millennial climate activity, are consistent with a memory-less stationary random process. From the data at hand we cannot exclude the possibility that the long-term variations occurred by chance. If we however divide a DO cycle into two independent processes governing warming and cooling, this is not true anymore and significant time-varying structure is detected. We thus propose a model that incorporates long-term variations through forcing of the parameters with external climate factors. We find good agreement with the data in a model where the mean duration of interstadial phases of the DO cycle is controlled by global ice volume and the stadial phases by boreal summer insolation. This finding can help to differentiate the mechanisms proposed to cause DO events.

Data availability. The principle data that are used are the timings reported in a table in Rasmussen et al. (2014) (cited in the article). The insolation data are publicly available as supporting online material to Huybers (2006) (DOI:https://doi.org/10.1126/science.1125249). The ice volume data from Lisiecki and Raymo (2005) are also publicly available at https://doi.pangaea.de/10.1594/PANGAEA.704257. The NGRIP time series displayed in Fig. 1 (although not used in the analysis) is available at www.iceandclimate.nbi.ku.dk/data/.
Competing interests. The authors declare that they have no conflict of interest.

Acknowledgements. This project has received funding from the European Union's Horizon 2020 research and innovation Programme under the Marie Skłodowska-Curie grant agreement no. 643073 .

Edited by: Luke Skinner

Reviewed by: Valerie N. Livina and Takahito Mitsui

\section{References}

Alley, R. B., Anandakrishnan, S., and Jung, P.: Stochastic Resonance in the North Atlantic, Paleoceanography, 16, 190-198, 2001.

Braun, H., Christl, M., Rahmstorf, S., Ganopolski, A., Mangini, A., Kubatzki, C., Roth, K., and Kromer, B.: Possible solar origin of the 1,470-year glacial climate cycle demonstrated in a coupled model, Nature, 438, 208-211, 2005.

Braun, H., Ganopolski, A., Christl, M., and Chialvo, D. R.: A simple conceptual model of abrupt glacial climate events, Nonlin. Processes Geophys., 14, 709-721, https://doi.org/10.5194/npg14-709-2007, 2007.

Braun, H., Ditlevsen, P., Kurths, J., and Mudelsee, M.: Limitations of red noise in analysing Dansgaard-Oeschger events, Clim. Past, 6, 85-92, https://doi.org/10.5194/cp-6-85-2010, 2010.

Buizert, C. and Schmittner, A.: Southern Ocean control of glacial AMOC stability and Dansgaard-Oeschger interstadial duration, Paleoceanography, 30, 1595-1612, 2015.

Dansgaard, W., Johnsen, S. J., Clausen, H. B., Dahl-Jensen, D., Gundestrup, N. S., Hammer, C. U., Hvidberg, C. S., Steffensen, J. P., Sveinbjörnsdottir, A. E., Jouzel, J., and Bond, G.: Evidence for general instability of past climate from a 250-kyr ice-core record, Nature, 364, 218-220, 1993.

Ditlevsen, P. D., Kristensen, M. S., and Andersen, K. K.: The Recurrence Time of Dansgaard-Oeschger Events and Limits on the Possible Periodic Component, J. Climate, 18, 2594-2603, 2005.

Ditlevsen, P. D., Andersen, K. K., and Svensson, A.: The DOclimate events are probably noise induced: statistical investigation of the claimed 1470 years cycle, Clim. Past, 3, 129-134, https://doi.org/10.5194/cp-3-129-2007, 2007.

Dokken, T. M., Nisancioglu, K. H., Li, C., Battisti, D. S., and Kissel, C.: Dansgaard-Oeschger cycles: Interactions between ocean and sea ice intrinsic to the Nordic seas, Paleoceanography, 28, 491502, 2013.

Drijfhout, S., Gleeson, E., Dijkstra, H. A., and Livina, V.: Spontaneous abrupt climate change due to an atmospheric blocking-seaice-ocean feedback in an unforced climate model simulation, $\mathrm{P}$. Natl. Acad. Sci. USA, 110, 19713-19718, 2013.

Grootes, P. M. and Stuiver, M.: Oxygen 18/16 variability in Greenland snow and ice with $10^{-3}$ to $10^{5}$-year time resolution, J. Geophys. Res., 102, 26455-26470, 1997.

Huybers, P.: Early Pleistocene Glacial Cycles and the Integrated Summer Insolation Forcing, Science, 313, 508-511, 2006.

Kawamura, K., Abe-Ouchi, A., Motoyama, H. et al.: State dependence of climatic instability over the past 720,000 years from 
Antarctic ice cores and climate modeling, Sci. Adv., 3, e1600446, https://doi.org/10.1126/sciadv.1600446, 2017.

Kleppin, H., Jochum, M., Otto-Bliesner, B., Shields, C. A., and Yeager, S.: Stochastic Atmospheric Forcing as a Cause of Greenland Climate Transitions, J. Climate, 28, 7741-7763, 2015.

Lisiecki, L. E. and Raymo, M. E.: A Pliocene-Pleistocene stack of 57 globally distributed benthic $\delta^{18} \mathrm{O}$ records, Paleoceanography, 20, PA1003, https://doi.org/10.1029/2004PA001071, 2005.

Mitsui, T. and Crucifix, M.: Influence of external forcings on abrupt millennialscale climate changes: a statistical modelling study, Clim. Dyn., 48, 2729, https://doi.org/10.1007/s00382-016-3235z, 2017.

Peltier, W. R. and Vettoretti, G.: Dansgaard-Oeschger oscillations predicted in a comprehensive model of glacial climate: A "kicked" salt oscillator in the Atlantic, Geophys. Res. Lett., 41, 7306-7313, 2014.
Rahmstorf, S.: Timing of abrupt climate change: A precise clock, Geophys. Res. Lett., 30, 1510, https://doi.org/10.1029/2003GL017115, 2003.

Rasmussen, S. O., Bigler, M., Blockley, S. P. et al.: A stratigraphic framework for abrupt climatic changes during the Last Glacial period based on three synchronized Greenland ice-core records: refining and extending the INTIMATE event stratigraphy, Quaternary. Sci. Rev., 106, 14-28, 2014.

Schulz, M.: On the 1470-year pacing of Dansgaard-Oeschger warm events, Paleoceanography, 17, 41-49, 2002.

Svensson, A. et al.: The Greenland Ice Core Chronology 2005, 15 42 ka. Part 2: comparison to other records, Quaternary. Sci. Rev., 25, 3258-3267, 2006.

Timmermann, A., Gildor, H., Schulz, M., and Tziperman, E.: Coherent Resonant Millennial-Scale Climate Oscillations Triggered by Massive Meltwater Pulses, J. Climate, 16, 2569-2585, 2003. 